\title{
Tendencias en las construcciones residenciales para mediados de la década de los 90 's
}

\author{
National Association of Home Builders (NAHB) ${ }^{1}$ \\ Traducido por Laura C. Ruelas Monjardín
}

RESUMEN

Este artículo de la National Association of Home Builders (NA HB) que se basa en una investigación de la industria y el gobierno de los EU, identifica algunos de los dramáticos desarrollos que están redimensionando la industria de la construcción residencial en los EU cuando el siglo XXI se vislumbra cercano. Explora sus implicaciones para la construcción y diseño de casas y sugiere formas por las que los constructores innovadores puedan continuar operando exitosamente en un ambiente comercial restringido. Debido a que frecuentemente las tendencias en la industria de la construcción residencial en los EU, son un precedente para las de otras partes del mundo, pensamos que los constructores de cualquier otra parte encontrarán este artículo de interés.

PALABRAS CLAVE:

Industria, construcción de casas, Estados Unidos, viviendas, mercadotecnia.

\section{NUEVO PANORAMA DEMOGRAFICO, NUEVO ESTILO DE VIDA Y DISEÑOS}

Un estudio del crecimiento en el tipo de vivienda en los EU de 1984 a 1994, publicado por la influyente revista American Demographics, revela que el

\begin{abstract}
Based on U.S. government and industry research, this article from the National Association of Home Builders (NAHB) identifies some of the dramatic developments that are re-shaping the home-building industry in the United States as the 21 st century draws near. It explores their implications for home design and construction and suggests ways in which innovative builders can continue to operate profitably in a constrained business environment. Since home-building industry trends in the United States often foreshadow those in other parts of the w orld, we think builders elsew here will find it of interest.
\end{abstract}

KEY WORDS:

Home-building, industry, United States, housing, marketing.

porcentaje de familias "normales" (casadas, con hijos menores de 18 años) se ha incrementado en menos del $2 \%$. Aunque en el mismo período se dieron incrementos de casi $45 \%$ en el número de viviendas habitadas por personas sin parentesco, $31 \%$ de padres solos, $25 \%$ de familia numerosa, $18 \%$ de 
una sola persona y $10 \%$ de matrimonios sin hijos. Parece que el concepto de "casa", quizá hasta el de "familia" se está redefiniendo y esto tiene implicaciones importantes para los constructores de casas.

No sólo están creciendo más rápidamente los tipos de familias notradicionales en comparación con la familia "nuclear" tradicional estadounidense, sino que también otros desarrollos socioeconómicos deben ser tomados en cuenta por los constructores de casas. Por ejemplo, más personas trabajan hoy en su propia casa. Algunos de ellos son profesionistas y administradores desplazados por la gran tendencia a la baja en el tamaño de los equipos humanos de las empresas en ese país, quienes ya no pueden encontrar un empleo similar al que tuvieron por años. En muchos casos, ellos han optado por crear consultorías o negocios comerciales que operan desde sus casas. Para ese propósito están equipando algunos espacios de la casa para convertirlos en oficinas, con computadoras, teléfonos modernos, copiadoras, faxes y otros accesorios de una oficina sofisticada moderna.

Otros se llevan a su casa trabajo de la empresa y manejan sus negocios en las tardes o durante los fines de semana. Algunos más están en la "telecomunicación", trabajando en casa y comunicándose continuamente con las oficinas de otras compañías vía teléfono, fax y módem. Todas estas personas estarán pensando en la forma de adaptar sus nuevos espacios de trabajo con mejor funcionalidad y eficiencia. En definitiva, muchos de ellos comprarán nuevas casas diseñadas especialmente para adaptarlas a sus necesidades de trabajo y pedirán a los constructores remodelar sus actuales casas para ese fin.
Impredeciblemente, la antigua familia numerosa, incluyendo los abuelos y los adolescentes, que desapareció en gran medida de los Estados Unidos durante las décadas posteriores a la Segunda Guerra Mundial está haciendo su regreso. Muchos abuelos están viviendo en familia, cuando ya no son capaces de valerse física y económicamente por sí solos, por lo que sus descendientes adultos los mantienen en sus últimos años de vida. Los hijos mayores, muchos de ellos con estudios profesionales, se están encontrando en la actualidad con que es difícil conseguir un trabajo adecuado y vivir de manera independiente, por lo que están regresando a vivir con sus padres. Pero la típica casa de una familia estadounidense sin hijos no está diseñada para la vida multigeneracional por lo que la familia ampliada está obligando a los constructores a diseñar casas para ese nuevo estilo de vida.

\section{ECONOMIA DE LA CLASE MEDIA}

Un estudio de Rutgers University subrayó las "desigualdades en el ingreso" que prevalecen entre la clase media de EU, el baluarte de la economía de la nación desde la Segunda Guerra Mundial. Medido en dólares de 1993, el ingreso promedio por familia en ese país se elevó $101.5 \%$, entre 1950 y 1973, aunque ha aumentado sólo $0.2 \%$ desde 1974 . El número de familias acomodadas se ha incrementado de alguna manera, aunque el promedio de familia está luchando por mantener su estilo de vida, al que se acostumbró por una generación o más. Así que cuando estas familias compren una casa nueva, irán dispuestos a encontrar una buena oferta de los constructores, para emplear su dinero de la mejor manera posible. 


\section{CONSTRUCCION E INDIVIDUALIDAD}

Irónicamente, debido a que su poder adquisitivo se contrae, los compradores de casas de la clase media están demandando más área construida e individualidad en sus nuevas casas y a precios más modestos. Por alguna razón, se han educado en el "servicio al cliente", tema muy de moda entre virtualmente todos los fabricantes y minoristas americanos en los últimos años. Si el anuncio sobre la calidad y satisfacción del cliente que hacen tan fehacientemente los constructores de casas es una patraña, la generación actual de compradores de casas está reclamando por ello. Por otra parte, si los compradores están renuentes a dar el salto hacia el nuevo mercado de casas, su paciencia y perspicacia en la compra y espera para la mejor casa y la mejor hipoteca para su dinero y el repetido incremento de la tasa de préstamo del Federal Reserve Bank deberán trabajar juntos para crear el mercado del comprador. Para mantenerse en los negocios y vender sus productos, los constructores tienen que acoplarse a las demandas de construcción, haciendo uso de tecnologías avanzadas para mantener márgenes de ganancia aceptables.

\section{CONCIENCIA AMBIENTAL}

A pesar de su desfalleciente poder adquisitivo, la mayoría de los consumidores americanos tienen una marcada conciencia ambiental cuando están a la búsqueda de una casa nueva. De acuerdo con M arvin Cetron, presidente de Forecasting International, en A rlington, Virginia, el aspecto ambiental se ha vuelto una característica permanente en el sistema de valores de muchos consumidores. Ellos quieren saber que el aire que respiran dentro de su casa no estará lleno de emisiones tóxicas de los materiales de construcción y de los acabados de la casa. Y muchos también quieren que sus casas sean diseñadas de manera tal que tengan el mínimo impacto ecológico en sus vecinos y en el ambiente natural.

Algunos constructores, como J ohn Clark de Washington, D.C., han adoptado la meta de la "sustentabilidad", una técnica de planeación en la que las áreas construíbles están en función de los recursos naturales y la vida silvestre del sitio, donde se utilizan sistemas y productos no tóxicos y donde la meta es la autosuficiencia de la comunidad. Clark es el diseñador de Haymount, Virginia, una nueva comunidad de 1700 acres en el Rio Rappahannock, situada a aproximadamente 50 millas al sur de Washington, D.C. El desarrollo residencial constará de 4,000 casas, así como oficinas y espacios para comercio. Las características de corte ecológico incluyen:

- Calles angostas, estacionamientos limitados y autobuses de enlace (shuttle buses) para limitar el uso del automóvil.

- Control del impacto de las tormentas utilizando pavimentos porosos y filtros de vegetación que absorban la escorrentía y estabilicen el suelo para reducir la erosión.

- Pozos verticales, poco profundos, que suministran agua potable mediante acequias horizontales que salen bajo el cauce del rio y dejan el río imperturbable.

- Una laguna para sedimentación y una serie de áreas húmedas construídas para tratar en el lugar, el agua que desecha la comunidad. 
- Un administrador del ambiente en el lugar, que vigila todo el reciclaje de materiales de construcción y otros, la disposición de los materiales peligrosos, la administración de las normas de uso de la energía y la asistencia técnica a los propietarios de tierras.

- Una granja orgánica, a cargo de un granjero independiente, que proporciona productos no tratados con químicos que producen los residentes de Haymount.

Aunque son relativamente pocos los compradores de casas que tendrán la oportunidad de acceder, en un futuro cercano, a una comunidad sustentable, como Haymount, muchos esperan que los constructores incluyan procesos de reciclaje. De acuerdo con un estudio reciente de la National Kitchen \& Bath Association, el $52 \%$ de los trabajos de diseño de cocinas incluyen actualmente la planeación de reciclado y el $37 \%$ ya incluye el reciclado de latas.

\section{ESPACIOS FLEXIBLES}

Los requerimientos de construcción actuales, con frecuencia incluyen planos flexibles que den a los compradores la opción de versatilidad en el uso de las habitaciones o espacios. Las familias que crecen porque cuidan a abuelos 0 a un adulto que no pueden vivir independientes, posteriormente reducen su tamaño cuando el primero muere o el otro encuentra un trabajo adecuado. Las oficinas en casa de los padres o el rincón para la computadora de los niños, cuyos usos pueden aumentar o desaparecer con el tiempo, crean demandas similares para los espacios flexibles. Los dueños de casas quieren poder rediseñar los espacios interiores, de manera que sirvan a sus actuales estilos de vida.
El Arq. Stephen Kendall de Tecnología + Economía en Mitchellville, Maryland, es uno de los líderes en el país que proponen "construcción abierta", un sistema que se adecúa a los espacios habitables diseñados por el cliente, en cascarones que soportan la carga con sistemas mecánicos fijos. Las divisiones, accesorios y acabados se ajustan al presupuesto y necesidades individuales de los compradores y pueden ser cambiados por los nuevos dueños 0 inquilinos." Mucha gente quiere decidir el diseño interior, los accesorios y los equipos," dice Kendall. "Ellos ya no quieren mudarse a casas diseñadas por otra persona".

Los centros de diversión familiares con grandes pantallas de t.v., sistemas multiconferencias en sonido de $C D$ e instalaciones para juegos electrónicos, se están volviendo las características principales de la vida de familias de la clase media en EU. Los constructores adoptan medidas sabias al ofrecer opciones que se acoplen a esta tendencia, desde muros a la mitad en el área familiar hasta plataformas para "teatro en la casa" en un espacio separado. Un estudio realizado por Electronic Industries Association en EU, indicó que el $56 \%$ de los consumidores consideraron la preinstalación de teatro en la casa como una opción atractiva para una nueva casa.

Los espacios adicionales para vivir, tales como un apartamento con una entrada privada, construída sobre un estacionamiento aparte, son maneras sensatas de alojar a los abuelos, un adolescente $o$ un inquilino. Tienen la doble virtud de añadir el espacio necesario para vivir y permitir un grado de independencia con el que no se cuenta habitando bajo un techo común. Muchos constructores estarán suministrando a estos apartamentos, estudios hasta donde el 
espacio y los reglamentos para viviendas lo permitan.

\section{OPCIONES DE ESTILOS DE VIDA}

Otros espacios domésticos que se están redefiniendo debido a las nuevas tendencias demográficas y socioeconómicas son la cocina y el comedor. "La cocina es el nuevo cuarto familiar", dice J oan McCloskey, editor de la revista Better homes and gardens. Es el nuevo espacio donde los miembros de la familia aprovechan para comentar las actividades del día mientras preparan los alimentos. Es el sitio donde se come y donde los niños hacen su tarea, por lo que actualmente, el diseño de las cocinas contemplan espacios para computadoras para hacer tareas y preparar el menú.

Dependiendo de los estilos de vida de cada familia, el comedor formal puede ser una cosa del pasado o un santuario para valores tradicionales. Debido a que hasta en las familias tradicionales, apenas sí se usa, este espacio con frecuencia se elimina para crear un diseño más económico o utilizarse para diferentes usos. Aunque en una parte de la población perduran lazos psicológicos con el pasado, como un baluarte contra la violenta corriente moderna del continuo cambio y uno de esos lazos puede ser el comedor formal. Lo mismo pasa con otros diseños "tradicionales" o "conservadores".

A todas luces, los constructores deben entender los estilos de vida para los que están construyendo, porque ya no es posible ofrecer un solo diseño que se ajuste a todos los intereses. Existen otras tendencias, producto de una investigación de NAHB: el deseo de aplicaciones de alta tecnología que ahorre tiempo a los padres que trabajan; énfasis en lo atractivo, productos prácticos sin mucho brillo y chispa; y un interés en sistemas de seguridad automatizado que impidan los delitos y que incrementen el sentido de seguridad en la casa.

\section{LA RESPUESTA DE LA INDUSTRIA DE LA CONSTRUCCION DE CASAS}

Además de los retos en el diseño que presentan los cambios demográficos y estilos de vida, están las restricciones económicas que representan el alza de los costos de los materiales y las presiones regulatorias. Todas estas fuerzas empujan a los fabricantes a desarrollar nuevos productos de construcción y a los constructores a aceptar nuevas técnicas a un paso más rápido. Para evaluar la respuesta de la industria de la construcción de casas, el Centro de Investigación de NAHB, financiado por Masco Corporation, preguntó a más de 200 fabricantes, universidades, laboratorios y agencias gubernamentales en EU, en qué nuevos proyectos estaban trabajando. Los resultados aparecieron en el artículo "Tecnología en la construcción" en la edición de julio de 1994 de la revista Builder. Se resumió de manera selectiva en lo siguiente. La crisis por la que atraviesan los materiales en la industria de la construcción de casas en los EU yace en la disponibilidad y precio de la madera clasificada para elementos estructurales. La política de conservación de los recursos, aspectos ecológicos y prácticas en la industria de la construcción mantendrán impredecibles los precios de la madera en el futuro cercano, por lo que más y más constructores considerarán usar el acero, el concreto y los productos de madera de alta ingeniería para incrementar la estabilidad en el precio del mercado y mantener el valor para sus clientes. 


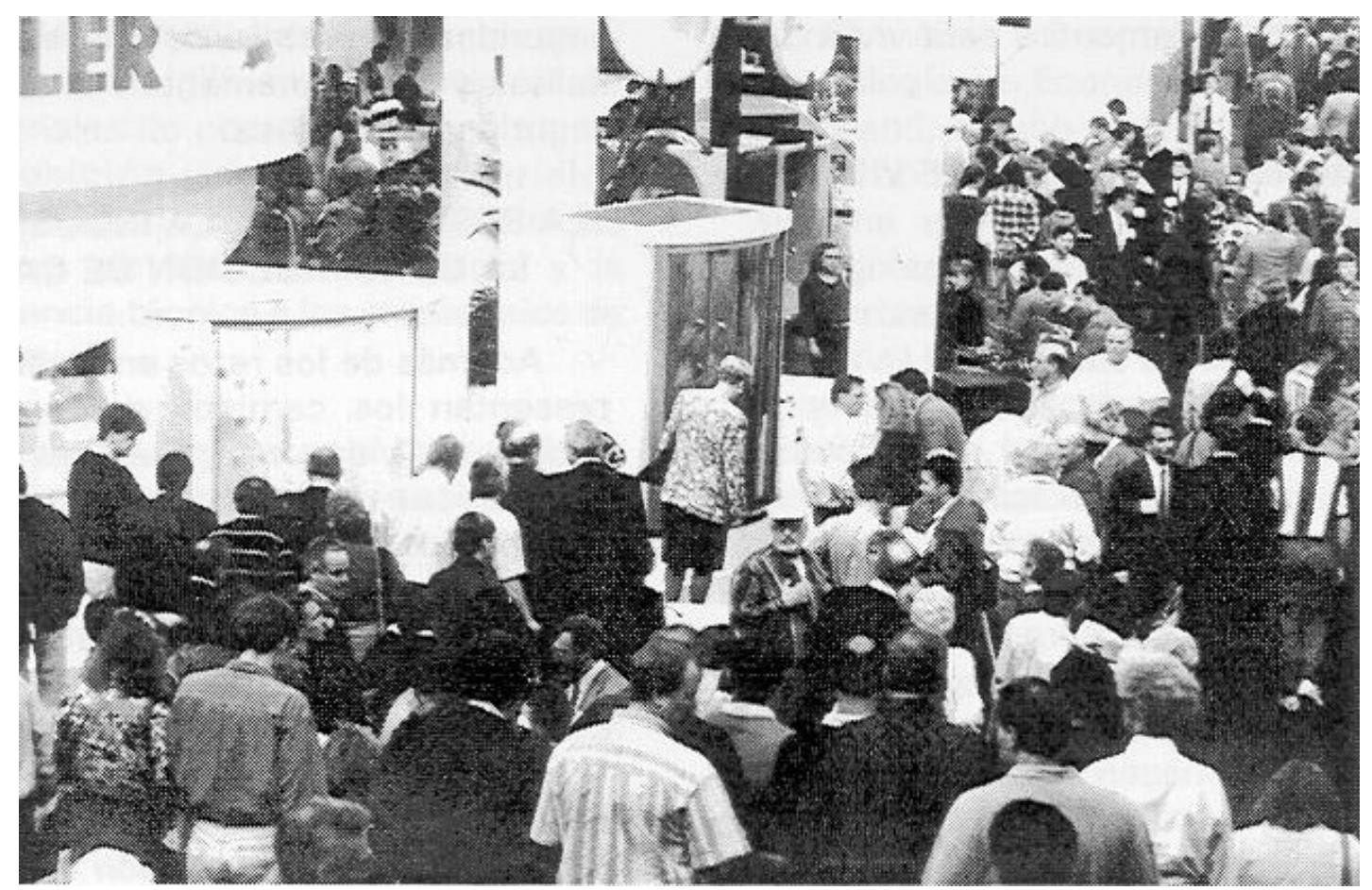

Exposición de los Constructores, Houston, Texas del 26 al 29 de Enero de 1996 con la asistencia de cerca de 70,000 profesionales de la construcción de casas-habitación

\section{Acero estructural de tamaño ligero}

- Debido a que los elementos de acero vienen en una amplia variedad de formas y tamaños, HUD (Departamento de Desarrollo Urbano y Vivienda de los EU) y NAHB han fundado el Centro de Investigación de NAHB para desarrollar normas, nomogramas de diseño y tablas de claros para elementos ligeros. El Centro también está evaluando el comportamiento térmico y acústico de las casas con elementos ligeros de acero para el Instituto Americano del Acero y Hierro.

- Para vencer la resistencia de los constructores con madera hacia el uso de elementos ligeros, por un sentimiento de aversión a lo que no sea madera, HL Stud Corporation ha desarrollado pies derechos de acero que se clavan a placas de madera. Los pies derechos no estructurales se utilizan para divisiones interiores.

- Los Sistemas Tri-Chord han cambiado la forma de los elementos de acero por medio de miembros triangulares y componentes de armaduras que se ajustan en el extremo superior en forma de $\mathrm{C}$ y en las placas inferiores.

\section{Sistemas innovadores en mampostería y concreto}

- La empresa General Shale ha desarrollado un tabique estructural para uso residencial con dos grandes perforaciones en donde se colocan las varillas de refuerzo y la mezcla de cemento. Los muros de mampostería reforzadoa de un sólo espesor eliminan la necesidad de elementos ligeros de madera, dice el vocero de la compañía Dennis Fair. También fabrican tabiques especiales para 
uniones con vigas y para aberturas de puertas y ventanas.

- Hebel Southeast promueve el concreto celular esterilizado para la construcción de toda la casa, incluyendo sistemas de techo y piso, dice el director técnico Mike Ibrahim. Hebel hace bloques, paneles y hasta escaleras premoldeadas con este tipo de material.

- Concept Homes ha desarrollado un nuevo sistema para construir toda la casa, sin componentes de concreto premoldeado. El sistema incluye paneles para la cimentación, pisos suspendidos, R-36 (norma de aislamiento), muros exteriores $y$ techos R-36. La compañía dice que el tiempo típico de construcción es de menos de dos días y que el costo se compara con la construcción de elementos ligeros de madera en muchas áreas.

\section{Ingeniería de productos de madera}

- John B. Fery, Director de Boise Cascade, la segunda industria más grande productora de madera laminada a base de chapa, dijo que el mercado para los productos de madera de carácter ingenieril, está creciendo más rápido que el de productos de madera convencionales. Como evidencia, él señala que su compañía vendió $\$ 71$ millones de dólares de esos productos en 1993, $87 \%$ más que en 1992.

- Investigadores del U.S. Forest Service, Forest Products Laboratory, en Madison, Wisconsin, están buscando las formas de hacer un uso más eficiente de las diferentes calidades y especies de madera, incluyendo las angiospermas de más baja calidad para "laminados encolados".

- El Forest Products Laboratory, University of West Virginia y
Massachusetts Institute of Technology están trabajando buscando métodos para rigidizar las vigas laminadas y las vigas I de madera con plásticos reforzados con fibras.

El mismo tipo de experimentos lo están realizando organizaciones privadas y gubernamentales sobre materiales innovadores $y$ sistemas para cimentaciones, exteriores, ventanas y puertas, plomería, HVAC (calefacción, ventilación y aire acondicionado), dispositivos, herramientas y cualquier otro aspecto de la construcción de casas. La industria de la construcción de casas de los EU está investigando todas las "vías" para satisfacer de manera simultánea la demanda del mercado y los requerimientos de los reglamentos.

\section{NUEVOS METODOS DE ADMINISTRACION DE PROYECTOS Y MERCADOTECNIA}

Keith Brow n, presidente de Sun Forest Construction, no es un típico constructor de casas. El construye casas por más de 450,000 dólares, dentro de un radio de 2 millas en Chapel Hill, North Carolina, Aunque personalmente ve su compañía diseño y construcción como un laboratorio para toda la industria de la construcción de casas-habitación. Dice, que los sistemas que actualmente está desarrollando en su taller, serán comunes en cinco años. "La forma que construimos y hacemos negocios va a cambiar debido a la computadora", dice Brown, quien también es co-propietario de UFAB, una compañía que produce BUILDSOFT, un sistema de software especial para la construcción.

"Podemos estimar con precisión una casa cuando todavía es un concepto", dice Brown. "Lo que significa que podemos hacer cualquier cambio que el cliente quiera y que conozca nuestros costos a lo largo del proceso". El sistema 
de despegue de Brown almacena y actualiza automáticamente los precios al vendedor y puede seleccionar a los vendedores con base en el precio y la preferencia. La programación se integra con la estimación y la cantidad de materiales necesaria para construir una casa y el proceso de construcción en papel. El sistema utiliza un método de ruta crítica, el cual Brown ajusta para acomodar las características de cada casa. Una vez que el programa y las cantidades están completas, el sistema de Brow $n$ automáticamente crea las órdenes de adquisiciones para enviarlas a los proveedores y a los subcontratistas. El estima que la automatización de los programas y órdenes de adquisición pueden añadir cuando mucho el $10 \%$ al precio más bajo del constructor.

El superintendente de Brow n controla la construcción utilizando una computadora lap-top Pentium en el lugar de construcción. Cada noche, desde su casa, el superintendente automáticamente baja la información reciente a su sistema principal via módem telefónico, lo que permite a Brown mantener un control firme en sus proyectos. "La computarización permitirá a los pequeños constructores estar al mismo nivel que los grandes", dice Brown. De hecho, él cree que la gente prefiere tratar con el pequeño constructor, quién está sistematizado y organizado para competir con los más grandes y más impersonales compañías.

Con cada nuevo reto al ingenio de los constructores de casas, se presentan nuevas oportunidades de ganancias. Los diferentes segmentos de mercado creados por las condiciones socioeconómicas y demográficas, ya mencionadas, significan una oportunidad para los pequeños constructores que son astutos con la computadora al tener una posición como especialistas en ese nicho y dar atención personalizada a los compradores. Los diseños distintivos de casas y los programas de mercadotecnia se pueden enfocar a padres solteros, parejas jóvenes sin hijos, familias jóvenes, familias maduras que se están mudando, jubilados activos, asilos para jubilados 0 a una docena de nichos de mercado bien definidos.

Pero hay que ser conscientes: su mercadotecnia no debe identificar hoy a compradores independientes como un estereotipo de nicho. Los vendedores necesitan tratar a cada prospecto como un individuo y los constructores necesitarán fabricar no sólo el producto, sino toda la experiencia de compra de casa. La consulta creciente de los consumidores a las fuentes de información en línea, implicará que los constructores a veces se anunciarán en el ciberespacio. Será más barato que anunciarse en los periódicos, y los constructores podrán capturar información de perspectiva sobre el inicio de proceso de compra de casa. Las oficinas de ventas harán un mejor uso de la tecnología de la computación lo que permitirá a los consumidores diseñar su casa, por ejemplo, mediante la automatización del proceso de selección de la opción o la sustitución. Aunque el hardware más poderoso y el software más inteligente nunca sustituirá la atención personalizada. Cuando mucho, el servicio excelente al cliente, distinguirá a aquellos constructores, pequeños 0 grandes, que tienen éxito en el mercado emergente de construcción de casas. 